\title{
Zur Wirksamkeit pharmakologischer und psychotherapeutischer Therapien bei Aufmerksamkeitsdefizit-/Hyperaktivitätsstörung (ADHS) im Erwachsenenalter: Eine empirische Metaanalyse
}

\author{
Friedrich Linderkamp ${ }^{\mathrm{a}}$ Gerhard Lauth ${ }^{\mathrm{b}}$ \\ ${ }^{a}$ Carl von Ossietzky Universität Oldenburg,

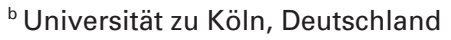

\section{Schlüsselwörter}

ADHS - Erwachsenenalter - Metaanalyse .

Therapieforschung · Pharmakotherapie

\section{Zusammenfassung}

Verlaufsstudien über die Aufmerksamkeitsdefizit-/Hyperaktivitätsstörung (ADHS) dokumentieren eine Persistenz von Kardinalsymptomen in ca. 1/3 der Fälle bis ins Erwachsenenalter. Somit stellt sich die Frage der Wirksamkeit der gängigen ADHS-Therapien für Erwachsene. Zur Beantwortung dieser Frage werden die Ergebnisse einer empirischen Metaanalyse vorgestellt. Einbezogen wurden 12 Wirksamkeitsstudien über psychotherapeutische Interventionen sowie 43 pharmakologische Studien. Im Ergebnis wurde für die psychotherapeutischen Studien eine gewichtete gemittelte Effektstärke von 0,84 (Konfidenzintervall 95\%: 0,64-1,04) berechnet und für die pharmakologischen Behandlungsformen eine gewichtete gemittelte Effektgröße von 0,44 (Konfidenzintervall 95\%: 0,37-0,50). Die Ergebnisse werden kritisch, unter Berücksichtigung methodischer (z.B. Einsatz unterschiedlicher Messinstrumente, unterschiedliche abhängige Variablen) und weiterführender inhaltlicher Gesichtspunkte, diskutiert.

\section{Einführung}

Längsschnittstudien belegen, dass die Aufmerksamkeitsdefizit-/Hyperaktivitätsstörung (ADHS) eine über das Kindesalter hinaus persistierende Störung ist. Bei etwa $40 \%$ der

\section{Keywords}

ADHD - Adulthood - Meta-analysis .

Therapy research · Pharmacotherapy

\section{Summary}

Efficacy of Pharmacological versus Psychotherapeutic Therapies in Adults with Attention Deficit/Hyperactivity Disorder (ADHD): An Empirical Meta-Analysis

Longitudinal studies on attention deficit/hyperactivity disorder (ADHD) show a persistency of the disorder until adulthood in up to $1 / 3$ of cases. This raises the question about the efficacy of the common therapies for this group. To answer this question, this article documents the results of an empirical meta-analysis, including 12 psychotherapy studies and 43 pharmacological studies. The results show a weighted effect size for the psychotherapeutic studies of 0.84 (confidence interval 95\%: 0.64-1.04). For the pharmacological studies, a weighted effect size of 0.44 (confidence interval 95\%: 0.37-0.50) could be found. The results are critically discussed, regarding methodological issues (e.g. usage of different instruments, different dependent variables) and further subject-related aspects.

\section{KARGER \\ Fax +497614520714 \\ Information@Karger.de}

www.karger.com

\section{(C) 2011 S. Karger GmbH, Freiburg}

$1016-6262 / 11 / 0214-0229 \$ 38.00 / 0$

Accessible online at:

www.karger.com/ver
Betroffenen bleiben Symptome bis ins Erwachsenenalter erhalten. Demzufolge sind über 3\% der Erwachsenen von ADHS betroffen [Fayyad et al., 2007; Vorstand der Bundesärztekammer, 2005]. Schätzungsweise $1 / 3$ von ihnen weisen zudem komorbide Störungen auf, wobei hauptsächlich Major 
Depression (9-19\%), Dysthymia (5-25\%), Bipolare Störung (2-19\%), Angststörungen (12-16\%), Alkoholmissbrauch (5-18\%), Cannabisabhängigkeit (etwa 14\%) und geringe Selbstbeherrschung (etwa 20\%) zu verzeichnen sind [Barkley et al., 2008; Miller et al., 2007; zusammenfassend Lauth und Minsel, 2009]. Zudem ist ein vermehrtes Vorkommen dissozialer Persönlichkeitsstörungen festzustellen [Sobanski und Alm, 2004].

Erwachsene mit ADHS zeigen ein fehlerhaftes und flüchtiges Arbeitsverhalten, ein hohes Maß an Vergesslichkeit, schlechtes Zeitmanagement, geringe Konzentrationsfähigkeit und leichte Ablenkbarkeit sowie geringe Ausdauer bei der Bewältigung von längeren Arbeitsvorhaben. Auch werden bevorstehende Aufgaben häufig mehrmals aufgeschoben, bevor mit der Bearbeitung begonnen wird, oder es werden Entscheidungen übereilt getroffen. Stimmungsschwankungen, eine schwache Frustrationstoleranz und ein oft geringes Selbstwertgefühl machen einen großen Teil des Leidensdruckes Betroffener aus [Barkley et al., 2008]. Es herrscht Einvernehmen darüber, dass die Problematik durch eine mangelnde Selbststeuerung zu erklären ist. Die Gründe dafür werden in einem multifaktoriellen Bedingungsmodell zusammengefasst. Darin werden biologische, soziale und psychische Bedingungen aufeinander bezogen [Lauth und Raven, 2009; Schmidt und Petermann, 2009]. Als biologische Bedingungsfaktoren werden hauptsächlich eine grundlegende biologische Vulnerabilität, mangelnde Aktivierungssteuerung, Mängel in der zentralnervösen Informationsverarbeitung und Mängel in der Inhibitionskontrolle genannt. Unter Mitwirkung ungünstiger sozialer Umstände [Laucht et al., 2007] entstehen Funktionseinschränkungen (etwa eingeschränkte exekutive Kontrolle, unzureichende Gedächtnissysteme, unzureichendes metakognitives Kontrollsystem), die Lebensvollzüge und Alltagshandeln deutlich beeinträchtigen. So hat die Arbeitsgruppe um Barkley in einer 2008 vorgelegten Längsschnittstudie belegt, wie aus anfänglichen Verhaltensschwierigkeiten weitreichende Funktionseinschränkungen und soziale Anpassungsstörungen entstehen können [Barkley et al., 2008]. Die derzeitigen Studien zum Erscheinungsbild der ADHS schildern die Störung folglich vor allem als eine Alltagsbeeinträchtigung. Wichtige Kompetenzen (soziale Fertigkeiten, exekutive Kontrolle, Planungsverhalten) werden entweder nicht hinreichend gut gelernt (Kompetenzdefizit) oder nur eingeschränkt praktiziert (Performanzdefizit).

Auf der Grundlage dieses Bedingungsmodells lassen sich zwei Behandlungszugänge ableiten: 1.) die Verbesserung der zentralnervösen Informationsverarbeitung durch Psychopharmaka; 2.) die Verbesserung der Funktionsfähigkeit durch Übungsverfahren (Training), Coaching und Verhaltensberatung im Rahmen einer kognitiven Verhaltenstherapie. In der Praxis erfolgt oft eine Kombination beider Interventionen.

Beide Ansätze wurden bisher nicht auf ihre Wirksamkeit miteinander verglichen. $\mathrm{Zu}$ den psychologischen Therapien liegt bisher noch keine systematische Metaanalyse vor.
Die pharmakologische Interventionsstrategie gründet auf dem Postulat einer Störung im Katecholaminstoffwechsel. Eine katecholaminerge Dysfunktion im Frontallappen bzw. ein Ungleichgewicht von Konzentration und Bindungsfähigkeit verschiedener Neurotransmitter führt laut dieser Hypothese zu einer Regulationsstörung der Neurotransmittersysteme, insbesondere des dopaminergen Transmittersystems [Halperin und Schulz, 2006]. Hier greifen regulierend Medikamente ein. Es gibt eine Reihe von Medikamenten mit sehr unterschiedlichen Wirkstoffen. Speziell für ADHS-Betroffene im Erwachsenenalter fehlen für die meisten jedoch Wirksamkeitsnachweise. Dabei galt retardiertes und unretardiertes Methylphenidat aufgrund zahlreicher einzelner Untersuchungen, die die Effektivität des Wirkstoffes auch bei Probanden im Erwachsenenalter zeigen konnten, als wirksamstes Mittel. Seit April 2011 ist denn auch ein Methylphenidat-Medikament zur Behandlung Erwachsener mit ADHS in Deutschland zugelassen. Andere Wirkstoffe werden nur zurückhaltend empfohlen, da zum einen wenig Wirksamkeitsnachweise bestehen und zum anderen auch zum Teil starke Nebenwirkungen zu verzeichnen sind [Ebert et al., 2003; Vorstand der Bundesärztekammer, 2005]. Unter Berücksichtigung der Anzahl internationaler Wirksamkeitsstudien sind folgende Wirkstoffgruppen im Kontext der Pharmakotherapieforschung bei ADHS im Erwachsenenalter besonders relevant:

Methylphenidat: Die Wirkweise, wie Methylphenidat die ADHS-Symptome reduziert, ist noch nicht vollständig geklärt. Volkow et al. [2004] vermuten, dass die intrasynaptische Erhöhung von Noradrenalin und Dopamin im frontalen Cortex dafür verantwortlich ist.

Atomoxetin: Atomoxetin blockiert im zentralen Nervensystem den präsynaptischen Noradrenalintransporter, wodurch in den Nervenzellen die Wiederaufnahme von Noradrenalin verhindert wird [Simpson und Plosker, 2004]. Aufgrund seiner langen Wirkungsdauer wird Atomoxetin in der Regel nur einmal täglich eingenommen [National Collaborating Centre for Mental Health, 2009]. Anders als beim Methylphenidat besteht keine Missbrauchsgefahr [Barton, 2005]. Als Nebenwirkungen können dennoch vorübergehend Abdominalschmerzen, Schwindel, Übelkeit, Erbrechen, Gewichtsverlust durch verringerten Appetit sowie Erhöhung der Herzrate und des Blutdrucks auftreten [Wolraich et al., 2007].

Bupropion: Bupropion ist ein selektiver Wiederaufnahmehemmer von Noradrenalin und Dopamin und gehört zu den atypischen Antidepressiva. Es wurde ursprünglich zur Behandlung der Major Depression sowie zur Unterstützung der Raucherentwöhnung entwickelt [Wilens et al., 2005]. Zu den Nebenwirkungen gehören Mundtrockenheit, Fieber, Halluzinationen, Kopfschmerzen, Schlaflosigkeit und Konzentrationsstörungen [British Medical Association und The Royal Pharmaceutical Society of Great Britain, 2007].

Dexamphetamin: Dexamphetamin ist wie Methylphenidat ein Noradrenalin- und Dopaminwiederaufnahmehemmer. Zusätzlich hinterlässt es aber auch Noradrenalin und Dopa- 
min im extraneuralen Raum, indem der intraneurale vesikuläre Monoamintransporter blockiert wird. Zu den Nebenwirkungen zählen Mundtrockenheit, Schlafstörungen, Gewichtsverlust sowie als Langzeitfolgen Magenschmerzen, verminderter Appetit und Psychosen [National Collaborating Centre for Mental Health, 2009].

Atomoxetin, Bupropion und Dexamphetamin sind in Deutschland zur Behandlung der adulten ADHS nicht zugelassen.

Die psychotherapeutische Behandlung orientiert sich in der Regel am kognitiv-behavioralen Ansatz, der die ADHS-typischen Handlungsbeeinträchtigungen bzw. Kompetenzdefizite in den Blick nimmt. Aufbauend auf einer ausführlichen Psychoedukation werden spezifische Kompetenzen (Gedächtnis, Kommunikation, exekutive Kontrolle, Problemlösen) eingeübt und Voraussetzungen für eine bessere Selbstorganisation (Tagesstruktur, Terminkalender, Haushaltsführung, Geldeinteilen) geschaffen. Zudem werden Techniken vermittelt, mit denen Betroffene besser mit ihrer Störung umgehen lernen und Symptomreduktionen sowohl im Bereich der Kardinalsymptomatiken als auch in Bezug auf komorbide Störungen erreicht werden sollen.

\section{Methodik}

\section{Literaturrecherche}

In einem ersten Schritt wurde eine Literaturrecherche durchgeführt; dabei wurden die Literaturdatenbanken PsycINFO, Psyc Index sowie Google Scholar vom 1. Quartal 2010 ausgehend rückblickend durchsucht, um relevante Untersuchungen zu pharmakologischen und psychotherapeutischen Behandlungen der ADHS im Erwachsenenalter zu finden.

Es fanden sich insgesamt nur wenige Studien zu psychotherapeutischen Behandlungsmaßnahmen. Da andernfalls die Anzahl der Psychotherapiestudien für statistische Effektstärkenberechnungen nicht ausreichend gewesen wären, wurden sowohl Interventionen mit Kontrollgruppen als auch solche mit lediglich Prä-post-Messungen einbezogen. Somit konnten insgesamt 12 Studien ermittelt werden, wobei lediglich bei 4 Untersuchungen ein Kontrollgruppen-Design vorlag, wohingegen bei den anderen 8 Studien ausschließlich eine Interventionsgruppe vorhanden war.

Bei den pharmakologischen Untersuchungen wurde als Einschlusskriterium definiert, dass in den jeweiligen Studien entweder durch ein Kontrollgruppen- oder durch ein Cross-over-Design eine Placebo-Bedingung geschaffen worden sein musste. Beim Cross-over-Design werden die Probanden in 2 Gruppen aufgeteilt. Die erste Gruppe erhält dann zuerst die Medikation A und danach die Medikation B, die zweite Gruppe erhält zuerst die Medikation B und anschließend die Medikation A. Wenn nun bei beiden Gruppen derselbe Unterschied nachgewiesen wurde, ist dieser Unterschied evident.

Dabei fanden sich insgesamt 22 Studien mit Parallel-Gruppen-Design und 21 Cross-over-Untersuchungen. Ausgeschlossen wurden also solche Studien, die keine Kontrolle der Interventionseffekte durch PlaceboBedingungen beinhalteten, sowie Untersuchungen, bei denen die Angabe der Untersuchungsergebnisse unzureichend war, um daraus Effektgrößen zu berechnen.

Insgesamt konnten bis zum Zeitpunkt der Literaturrecherche im 1. Quartal 2010 lediglich 12 Studien gefunden werden, die die Wirkung von psychotherapeutischen Interventionen bei ADHS im Erwachsenenalter überprüfen. Diese sind alle ab 1999 publiziert worden und dem kognitiv-behavioralen Paradigma zuzuordnen. Mit Ausnahme der Untersu- chung von Zylowska et al. [2008] wurde in allen Untersuchungen versucht, den Probanden Verhaltensstrategien zu vermitteln, um Alltagsschwierigkeiten im Privat- und Berufsleben besser bewältigen zu können. Deutliche Unterschiede ergeben sich in der Anzahl der Sitzungen, die von 4 Sitzungen à 1,5 h [Wiggins et al., 1999] bis zu 36 Sitzungen ohne Zeitangabe [Wilens et al., 1999] schwankt. Zudem beschränken sich 8 Studien auf reine Prä-post-Vergleiche einer Interventionsgruppe, wohingegen lediglich 4 Studien die Interventionserfolge mit einer Kontrollgruppe abgeglichen haben. Im Einzelnen wurde in den Studien wie folgt vorgegangen:

Wiggins et al. [1999]: 9 Personen mit einem Durchschnittsalter von 42 Jahren erhielten 4 Sitzungen Psychoedukation. In jeder Sitzung wurden einzelne Schwierigkeiten (z.B. Zeitmanagement) besprochen und Lösungs- bzw. Kompensationsansätze erarbeitet. Zudem wurden Hausaufgaben eingesetzt. Das Training erwies sich im Vergleich zu einer aus 8 Personen bestehenden Kontrollgruppe (Altersdurchschnitt 43 Jahre) als hoch wirksam in allen Zielbereichen (z.B. Hyperaktivität, Unaufmerksamkeit und emotionale Labilität).

Wilens et al. [1999]: 26 Personen mit einem Durchschnittsalter von 42,2 Jahren erhielten über etwa 1 Jahr hinweg im Umfang von 36 Sitzungen eine modifizierte Form der kognitiven Therapie nach Beck. 22 der Probanden erhielten im Untersuchungszeitraum auch eine Pharmakotherapie, deren Wirkung im Rahmen der Untersuchung aber nicht gesondert überprüft wurde. Auch litten einige der Probanden an komorbiden Störungen. Als Ergebnis der Studie stellte sich heraus, dass diese Probanden sowohl bezüglich ihrer ADHS-Symptomatik als auch in Bezug auf Angststörungen und Depressionen von der Therapie profitieren konnten.

Hesslinger et al. [2002]: In Anlehnung an die dialektische Verhaltenstherapie nach Linehan [1993], die für die Borderline-Störung entwickelt worden ist, erhielten 8 Personen eine Therapie über 13 Sitzungen à 2 h. Die Personen litten zum Teil zusätzlich zu ihrer ADHS-Problematik an komorbiden Störungen (Schlafstörungen, Depressionen, soziale Phobie). Sowohl bezüglich der ADHS-Symptomatik als auch in Bezug auf die untersuchten Komorbiditäten konnten Verbesserungen erzielt werden. Ein Vergleich zu einer Kontrollgruppe war trotz Parallel-GruppenDesign nicht möglich, da bei 7 Personen in der Kontrollgruppe 4 Dropouts zu verzeichnen waren.

Stevenson et al. [2002]: Es wurden 22 Personen (Durchschnittsalter 42 Jahre), die ein kognitives Förderprogramm zur Vermittlung von Selbstmanagementstrategien erhalten hatten, mit 21 Personen ohne Therapie (Altersdurchschnitt 36 Jahre) verglichen. Nach 8 Sitzungen à $2 \mathrm{~h}$ zeigten sich deutliche Verbesserungen der Experimentalgruppe im Vergleich zur Kontrollgruppe sowohl in Bezug auf die ADHS-Symptomatik als auch im Umgang mit Alltagsärger sowie bezüglich organisatorischer Fähigkeiten und Selbstvertrauen. Stevenson et al. [2002] schreiben, dass die Medikamentierung einiger Probanden keinen Einfluss auf die Untersuchungsergebnisse gehabt habe.

Safren et al. [2005]: 16 Versuchspersonen erhielten eine kognitive Verhaltenstherapie (ohne Angabe zur Länge und der Anzahl der Sitzungen). Bedingung für die Teilnahme an der Studie war, dass die Patienten bereits 2 Monate vor Therapiebeginn pharmakologisch behandelt worden sind und die Dosis während des letzten Monats um nicht mehr als 10\% abgeändert worden ist. Es wurde jedoch nicht differenziert erhoben, welche Pharmaka die Probanden eingenommen haben. Verglichen wurden diese Untersuchungsteilnehmer mit 15 Personen einer Kontrollgruppe, die ausschließlich mit Pharmaka behandelt worden sind. Als Resultat der Studie zeigte sich, dass die therapeutisch behandelten Personen sowohl stärker in Bezug auf ihre ADHS-Symptomatik als auch gemessen an ihrer Ängstlichkeit und ihrer Depression von der Intervention profitieren konnten. Die beiden Untersuchungsgruppen (Durchschnittsalter insgesamt 46 Jahre) unterschieden sich nicht signifikant in wesentlichen Merkmalen wie z.B. Geschlecht, Alter oder Stärke der Störung.

Rostain und Ramsey [2006]: 43 Personen im Durchschnittsalter von 31 Jahren wurden über 6 Monate kombiniert mit Amphetaminsalzen 
Tab. 1. Effektstärken psychotherapeutischer Studien

\begin{tabular}{|c|c|c|c|c|c|}
\hline Studie & n (ohne Drop-outs) & Kontrollgruppe & Effektgröße & Effektvarianz & $\begin{array}{l}\text { Konfidenzintervall }(95 \%) \\
\text { der Effektgröße }\end{array}$ \\
\hline Wilens et al. [1999] & 26 & nein & 0,91 & 0,18 & $0,07-1,75$ \\
\hline Wiggins et al. [1999] & 17 & ja & 0,92 & 0,30 & $-0,15-1,98$ \\
\hline Hesslinger et al. [2002] & 8 & nein & 1,67 & 0,94 & $-0,24-3,56$ \\
\hline Stevenson et al. [2002] & 43 & ja & 1,26 & 0,12 & $0,59-1,93$ \\
\hline Safren et al. [2005] & 31 & ja & 0,88 & 0,15 & $0,11-1,64$ \\
\hline Philipsen et al. [2007] & 66 & nein & 0,67 & 0,07 & $0,17-1,17$ \\
\hline Solanto et al. [2008] & 38 & nein & 1,15 & 0,13 & $0,44-1,86$ \\
\hline Virta et al.[2008] & 29 & nein & 0,23 & 0,15 & $-0,53-0,98$ \\
\hline Zylowska et al. [2008] & 23 & nein & 0,80 & 0,21 & $-0,09-1,69$ \\
\hline Bramham et al. [2009] & 78 & ja & 0,64 & 0,06 & $0,18-1,10$ \\
\hline Rostain und Ramsey [2006] & 43 & nein & 1,31 & 0,17 & $0,64-1,99$ \\
\hline Lauth et al. [2010] & 26 & nein & $\begin{array}{l}0,56 \\
\text { Homogenitätstest } \\
\text { bestanden }\end{array}$ & 0,12 & $-0,26-1,37$ \\
\hline Gesamt & 428 & & 0,84 & 0,01 & $0,64-1,03$ \\
\hline
\end{tabular}

und 16 Sitzungen à 50 min kognitiver Verhaltenstherapie behandelt. Bei Unverträglichkeit der Amphetaminsalze erhielten die Untersuchungsteilnehmer Methylphenidat. Es wurden kognitive Strategien erarbeitet, um beispielsweise ein besseres Zeitmanagement $\mathrm{zu}$ entwickeln. Neben der ADHS-Symptomatik wurden unter anderem auch die Depressivität, Ängstlichkeit und bipolare Affektivität mit erfasst. Insgesamt zeigten sich im Prä-post-Vergleich bedeutsame Unterschiede in allen Bereichen.

Diese Untersuchung ist die einzige, die explizit eine psychologischpharmakologische Kombinationsbehandlung untersucht.

Philipsen et al. [2007]: 66 Personen (Altersdurchschnitt 36 Jahre) erhielten eine strukturierte Gruppentherapie, die ein Trainingsprogramm zur Verbesserung der ADHS-Symptomatik beruhend auf verhaltenstherapeutischen und dialektisch-behavioralen Therapieprinzipien enthält. Die Intervention erfolgte über 13 Wochen hinweg mit wöchentlich je einer 2-stündigen Sitzung. Neben der Verbesserung der ADHS-Symptomatik konnten auch Erfolge bezüglich einer Reduktion der Depressivität und dem allgemeinen gesundheitlichen Status festgestellt werden.

Solanto et al. [2008]: Es wurde eine neue meta-kognitive Therapie erprobt, die auf den Prinzipien der Verhaltenstherapie beruht und speziell für die Behandlung von Erwachsenen mit einer ADHS entwickelt wurde. Dabei sollten neue Fertigkeiten im Umgang mit der ADHS-Symptomatik erlernt werden, um Alltagsschwierigkeiten besser bewältigen zu können. 12 Personen wurden hierbei über 8 und 26 über 12 2-stündige Sitzungen therapiert. Verschiedene Fragebögen zur ADHS-Symptomatik zeigten deutliche Verbesserungen hinsichtlich der Kardinalsymptome Unaufmerksamkeit, Impulsivität und Hyperaktivität. Komorbiditäten wurden in dieser Untersuchung nicht erfasst.

Virta et al. [2008]: 29 Personen zwischen 18 und 45 Jahren wurden mithilfe einer verhaltenstherapeutischen Gruppentherapie untersucht. Die Probanden wurden auf 4 Behandlungsgruppen aufgeteilt, wobei in jeweils 2 dieser Gruppen 10 bzw. 11 Sitzungen à 1,5-2 h stattgefunden haben. In jeder Sitzung wurden andere Defizite in Beruf und Alltag behandelt (z.B. Organisation, Gedächtnis, Impulsivität). Neben einer Verbesserung der ADHS-Symptomatik konnten auch Erfolge bezüglich der Depressivität festgestellt werden.

Zylowska et al. [2008]: 21 Erwachsene (Altersdurchschnitt 40 Jahre) und 4 Jugendliche mit einer ADHS erhielten über 8 Sitzungen, die jeweils 2,5 $\mathrm{h}$ andauerten, ein Achtsamkeits-Meditationstraining, dessen Ziel nicht Entspannung, sondern ein bewussteres Erleben von alltäglichen Gegebenheiten ist. Bezüglich der ADHS-Symptomatik konnte eine deutliche Verbesserung erzielt werden. Auswirkungen auf komorbide Störungen wurden nicht untersucht.

Bramham et al. [2009]: 41 Personen (Altersdurchschnitt 33 Jahre), die über 3 Monate hinweg jeden Monat an einem Workshop-Tag eine verhaltenstherapeutisch orientierte Gruppentherapie erhalten hatten, wurden mit 37 Personen (Durchschnittsalter 31 Jahre) einer Kontrollgruppe, die keine Intervention erhalten hatte, verglichen. Neben leichten Verbesserungen der Depressivität und Angst konnten die Probanden auch bezüglich ihrer Selbstachtung und ihres Selbstvertrauens profitieren. Die ADHS-Symptomatik wurde in dieser Untersuchung allerdings nicht gezielt kontrolliert.

Lauth et al. [2010]: Mithilfe eines kognitiv-behavioral orientierten Gruppentrainings wurden 26 Personen über 5 3-stündige Sitzungen mit dem Ziel der Reduktion ihrer ADHS-Symptomatik behandelt. Dabei wurden neben Hausaufgaben auch kursübergreifende Trainingsziele vereinbart. Die Probanden waren angehalten, die in dem Kurs erlernten Strategien zur Bewältigung von Alltagsschwierigkeiten im Alltag praktisch einzuüben. Sowohl für den Bereich der Unaufmerksamkeit als auch für den der Hyperaktivität konnten Verbesserungen erzielt werden. Nicht erfasst wurden komorbide Störungen.

Insgesamt muss die Studienlage als wenig zufriedenstellend bezeichnet werden. Abgesehen von kleinen Stichproben und wenigen randomisiert-kontrollierten Studien besteht kein standardisiertes Vorgehen bezogen auf die erhobenen Variablen. Während in einigen Studien nur ADHS-Symptome erhoben wurden, haben andere Studien auch komorbide Störungen mit erfasst.

\section{Durchführung der Metaanalyse}

Trotz der zahlreichen methodischen Mängel der pharmakologischen und der psychotherapeutischen Wirksamkeitsstudien ist zu erkennen, dass sowohl pharmakologische als auch psychotherapeutische Behandlungsformen wirksam sein können.

In der therapeutischen Praxis werden pharmakologische und psychotherapeutische Behandlungen oft in Kombination durchgeführt. In der Wirksamkeitsforschung wird diese Behandlungskombination jedoch nur in einer Studie von Rostain und Ramsey [2006] explizit untersucht. Daher wird in der vorliegenden Metaanalyse zunächst der Forschungsstand zur Wirksamkeit pharmakologischer und psychotherapeutischer Behandlungen jeweils separat erfasst und gegenübergestellt. Im Einzelnen wird überprüft, ob diese beiden Interventionsstrategien bedeutende Unterschiede aufweisen, indem die Studienergebnisse aus beiden Richtungen 


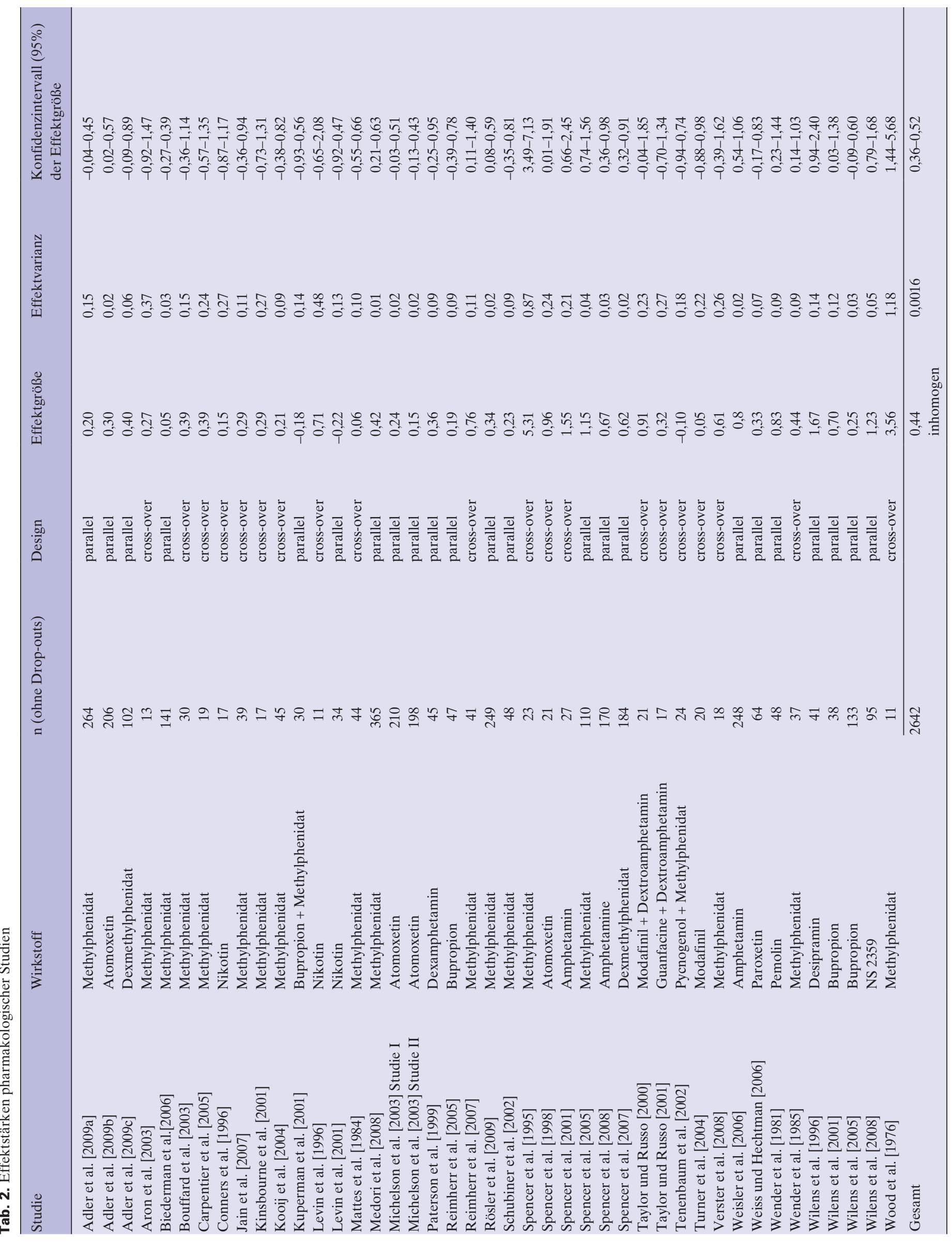


Tab. 3. Effektstärken pharmakologischer Studien mit gängigen und experimentell getesteten Wirkstoffen

\begin{tabular}{|c|c|c|c|c|c|}
\hline Medikament(engruppe) & $\begin{array}{l}\text { Anzahl Studien } \\
\text { (Versuchspersonen gesamt) }\end{array}$ & Effektgröße & Effektvarianz & $\begin{array}{l}\text { Konfidenzintervall } \\
\text { (95\% der Effektgröße) }\end{array}$ & Homogen? \\
\hline Methylphenidat & $22(1819)$ & 0,40 & 0,002 & $0,31-0,49$ & nein \\
\hline Bupropion & $4(218)$ & 0,28 & 0,019 & $0,01-0,54$ & ja \\
\hline Amphetamin & $6(511)$ & 0,74 & 0,008 & $0,56-0,92$ & ja \\
\hline Atomoxetin & $4(635)$ & 0,25 & 0,006 & $0,09-0,40$ & ja \\
\hline «Gängige» Wirkstoffe zusammen & $35(3183)$ & 0,41 & 0,001 & $0,34-0,48$ & nein \\
\hline Experimentell getestete Wirkstoffe & $11(330)$ & 0,66 & 0,013 & $0,43-0,88$ & nein \\
\hline
\end{tabular}

jeweils zusammengefasst und durch Ermittlung einer gemittelten Effektstärke verglichen werden.

Die Verrechnung der Daten erfolgte unter Nutzung der MicrosoftSoftware Excel 2007. Zur Berechnung der Effektstärken der einzelnen Untersuchungen wurde Cohens d mit gepoolten Varianzen verwendet. In Studien, die aufgrund der dargestellten Untersuchungsergebnisse keine eigene Berechnung der Effektgrößen ermöglichen, in denen aber Cohens $\mathrm{d}$ bereits angegeben worden ist, wurden die angegebenen Werte übernommen.

Die meisten Untersuchungen haben verschiedene Effektmaße als abhängige Variablen erfasst, die bei der Berechnung der Wirksamkeit gemittelt wurden. Daraus wurden - für die psychotherapeutischen und für die pharmakologischen Behandlungsformen getrennt voneinander gewichtete mittlere Effektstärken nach dem Prinzip der Summe der präzisionsgewichteten individuellen Effektstärken der Primärstudien berechnet [Rustenbach, 2003]. Berechnet wurde die mittlere Effektstärke also, indem die einzelnen Effektstärkenvarianzen bestimmt und deren Kehrwerte dann benutzt wurden, um das Gewicht der einzelnen Studien gemessen an allen Untersuchungsergebnissen zu bestimmen. Das heißt, es wurde jeweils der Varianzkehrwert der einzelnen Studie in Beziehung gesetzt zum Varianzkehrwert aller Studien, um so das Gewicht jeder einzelnen Studie am Gesamteffekt zu bestimmen. Als Produkt dieses Gewichts und der dazugehörigen Effektstärke wurde eine gewichtete Effektstärke bestimmt. Die Summe aller dieser gewichteten Effektstärken bildet die Grundlage für die gewichtete mittlere Effektstärke.

Zusätzlich wurden die zu den Studienergebnissen zugehörigen Konfidenzintervalle sowohl für die einzelnen Effektstärken als auch für den Gesamteffekt berechnet.

Auch wurde die Homogenität der integrierten Effekte mithilfe des Chi-Quadrat-Tests überprüft. Homogenität wird dabei als die Summe der gewichteten quadrierten Abweichungen der Studieneffekte vom mittleren Integrationseffekt verstanden [Rustenbach, 2003]. Ein signifikantes Ergebnis deutet daraufhin, dass die verschiedenen Studien nicht derselben Population angehören und die bestimmte gewichtete gemittelte Effektgröße in ihrer Aussagekraft eingeschränkt ist.

\section{Ergebnisse}

Für die psychotherapeutischen Studien wurde eine gewichtete gemittelte Effektstärke von 0,84 (Konfidenzintervall 95\%: 0,64-1,04) erzielt. Die Homogenitätsprüfung erbrachte eine Prüfgröße von 9,07, die aber den tabellarischen Chi-QuadratWert 19,68 für $\mathrm{df}=11$ bei einem Signifikanzniveau von $\alpha=$ $5 \%$ nicht überschreitet, womit das Ergebnis nicht signifikant ist und die Annahme der Homogenität der Studien als bestätigt gesehen werden kann. Detaillierte Ergebnisse der einzelnen Studien sind Tabelle $1 \mathrm{zu}$ entnehmen. Für die pharma- kologischen Behandlungsformen wurde eine gewichtete gemittelte Effektgröße von 0,44 (Konfidenzintervall 95\%: 0,37-0,50) erreicht (Tab. 2).

Wird statt der klassischen Metaanalyse eine Berechnung nach dem Random-Effect-Modell vorgenommen, zeigt sich auch bei Einbeziehung der zusätzlichen Varianzkomponente keine andere Ergebnislage: Die Effektstärke der pharmakologischen Studien liegt dann bei 0,41 (statt 0,44), jene der Psychotherapiestudien bei 0,82 (statt 0,84). Der tabellarische ChiQuadrat-Wert von ca. 58,124 wurde auf einem Signifikanzniveau von $\alpha=5 \%$ für $\mathrm{df}=42$ jedoch von der empirisch gefundenen Prüfgröße von 129,311 überschritten, weshalb das Ergebnis als signifikant und die Studien als inhomogen gelten müssen.

Da die mittlere Effektgröße bei Vorliegen eines inhomogenen Ergebnisses nur eingeschränkt interpretierbar ist, wurden 2 Subgruppen gebildet, die die Pharmakostudien aufteilen in Untersuchungen, die Wirkstoffe verwendet haben, die «gängig» sind, und solche, die lediglich experimentell in einzelnen Untersuchungen getestet wurden. Operationalisiert wurde letztere Kategorie dadurch, dass hier alle Wirkstoffe mit einbezogen worden sind, die in maximal 3 Untersuchungen Anwendung gefunden haben.

Auf dieser Basis wurden Methylphenidat $(\mathrm{n}=22)$, Bupropion $(\mathrm{n}=6)$, Atomoxetin $(\mathrm{n}=4)$ und die (gemischten) Amphetamine $(\mathrm{n}=4)$ als «gängige» Wirkstoffe klassifiziert, wobei eine Untersuchung die Wirkung von Methylphenidat und Bupropion miteinander vergleicht [Kuperman et al., 2001], sodass insgesamt in dieser Kategorie 35 verschiedene Studien Verwendung finden. Auf die restlichen Wirkstoffe entfallen insgesamt 11 Studien. 3 Untersuchungen, die 2 Wirkstoffe miteinander verglichen haben, die hier in beiden Kategorien verzeichnet sind, werden entsprechend auch in beiden Kategorien mit aufgezählt [Taylor und Russo, 2001; Tenenbaum et al., 2002].

Für die experimentell getesteten Studien ergab sich eine gewichtete mittlere Effektstärke von 0,66, wobei auch hier der Homogenitätstest aufgrund eines signifikanten Ergebnisses mit einer Prüfgröße von 237,867 nicht bestanden worden ist, da der kritische Chi-Quadrat-Wert von 18,307 bei $\alpha=5 \%$ somit deutlich überschritten wurde. Dies ist jedoch aufgrund der vielfältig eingesetzten Wirkstoffe an dieser Stelle nicht erwartungswidrig. 
Aber auch für die Untersuchungen mit «gängigen» Medikamenten ergab sich ein ähnliches Bild: Es wurde eine gewichtete mittlere Effektstärke von 0,41 erreicht (Tab. 3). Die kritische Grenze von 48,6 (bei $\alpha=5 \%$ und df =34) wurde jedoch von der Prüfgröße von 534,629 deutlich überschritten, sodass auch hier ein signifikantes und somit inhomogenes Ergebnis vorliegt.

Bei Betrachtung der einzelnen Wirkstoffe wird deutlich, dass auch für Methylphenidat allein betrachtet kein homogenes Ergebnis erzielt werden kann (Prüfgröße von 67,45 vs. Chi-Quadrat-Wert von 32,67 für df = 22-1), sodass die Möglichkeit einer Subgruppenbildung, die homogene Ergebnisse erzielt, unwahrscheinlich erscheint.

\section{Diskussion}

In die vorliegende Metaanalyse wurden 12 Wirksamkeitsstudien über psychotherapeutische Interventionen sowie 43 pharmakologische Studien einbezogen, die rückblickend vom 1. Quartal 2010 an publiziert wurden. Im Ergebnis wurde für die psychotherapeutischen Studien eine gewichtete gemittelte Effektstärke von 0,84 berechnet. Dies ist ein sehr hoher Effekt, der sich allerdings in der Hauptsache aus dem Vergleich von Prä-post-Erhebungen ergibt (8 von 12 Studien; nur bei 4 Studien wurden Vergleiche zu Kontrollgruppen hergestellt). Auf der Ebene dieser Vergleiche kann den psychotherapeutischen Interventionen beträchtliche Wirksamkeit und therapeutischer Nutzen bescheinigt werden. Die Interventionen verringern demnach sowohl die ADHS-Symptomatik als auch die Alltagsbeeinträchtigung.

$\mathrm{Zu}$ bedenken ist allerdings auch, dass die Probanden in 4 von 12 Studien zusätzlich zur Psychotherapie eine Medikation erhielten. Dies wurde nur in einer Studie methodisch kontrolliert und in einer weiteren Studie [Stevenson et al., 2002] kommen die Autoren zu der Feststellung, dass die Medikamentierung einiger Probanden keinen Einfluss auf die Untersuchungsergebnisse gehabt habe.

Für die pharmakologischen Behandlungsformen wurde eine gewichtete gemittelte Effektgröße von 0,44 errechnet. Das ist ein mittlerer Effekt, der aber im Vergleich zu Kontrollgruppen ohne Behandlung berechnet wurde.

Da die psychotherapeutischen und die pharmakologischen Studien eine unterschiedliche Methodenqualität aufweisen, kann man ihre Effektivität nicht vergleichend bewerten. Es fällt jedoch auf, dass die psychotherapeutischen Verfahren auch dann noch eine hohe Wirksamkeit zeigen, wenn man kontrollierte Studien (mit Kontrollgruppen-Design) heranzieht. 4 der insgesamt 12 aufgefundenen Studien [Wiggins et al., 1999; Stevenson et al., 2002; Safren et al., 2005; Bramham et al., 2009] hatten den Effekt der psychotherapeutischen Behandlungen gegen randomisierte Kontrollgruppen verglichen. In diesen 4 kontrollierten psychotherapeutischen Studien wird eine ebenfalls große durchschnittliche Effektstärke von
0,85 belegt; für die übrigen psychotherapeutischen Studien mit Prä-post-Vergleichen 0,82. Aufgrund der geringen Studienzahl kann dieses Ergebnis aber auch nicht vorbehaltlos interpretiert werden, sondern gibt bestenfalls einen hoffnungsvollen Trend wieder. Damit zeichnet sich nach der jetzigen, noch sehr unvollkommenen Befundlage eine beträchtliche Tauglichkeit der psychotherapeutischen Interventionen $\mathrm{ab}$.

Reflektiert man die mittleren Effekte der Pharmakostudien auf der Grundlage weiterer metaanalytischer Studien, zeigt sich folgendes Bild: In einer Metaanalyse von Faraone et al. [2004] konnte unter Einbeziehung von 6 Studien eine mittlere Effektstärke von 0,9 ermittelt werden. Positive Effekte stellten sich insbesondere bei hoher Dosierung $(\mathrm{d}=1,3)$ und unter Berücksichtigung des Medizinerurteils $(\mathrm{d}=1,1)$ ein. Wenn die Betroffenen selbst befragt wurden, errechnete sich allerdings eine Effektstärke von 0,03, womit keinerlei therapeutische Effekte konstatiert werden.

Unter Einbeziehung von 21 Studien fanden Faraone und Glatt [2010] signifikante Unterschiede in der Wirkung von lang wirkenden $(\mathrm{d}=0,73)$ und kurz wirkenden $(\mathrm{d}=0,96)$ Stimulantien versus Nicht-Stimulantien $(d=0,39)$. Auch in dieser Metaanalyse zeigten sich höhere Effektstärken bei Medizinerratings gegenüber dem Selbsturteil ( $d=0,63$ vs. $\mathrm{d}=0,43)$. Bei Studien, die Placebobedingungen mit kontrolliert hatten, zeigten sich gegenüber jenen, die darauf verzichtet hatten, deutlich geringere Effektstärken ( $d=0,36$ vs. $d=$ 0,75). Im Übrigen zeigten sich die Stimulantien-Responderraten mit $50 \%$ ( $20 \%$ bei Nicht-Stimulantien) niedriger als jene des Kindesalters (60-90\%, [Prosser und Reid, 1999]).

Schließlich bestätigen die Metaanalyse von Mészàros et al. [2009] unter Einbeziehung von 12 Studien $(d=0,67)$, die Metaanalyse von Koesters et al. [2008] unter Berücksichtigung von 16 Studien $(\mathrm{d}=0,42)$ sowie eine aktuelle Metaanalyse von Castells et al. [2011] mit 18 Studien $(d=0,58)$ die vorliegenden Ergebnisse, denn sie errechnen unter Placebobedingungen ebenfalls mittlere Effektstärken bei Methylphenidat-Medikation.

Insgesamt kann also davon ausgegangen werden, dass psychotherapeutische Interventionen den pharmakologischen Interventionen nicht nachstehen. Die vorliegenden Ergebnisse weisen sogar auf eine höhere Wirksamkeit hin. Diese höhere Wirksamkeit wäre angesichts der empirischen Befundlage zum Störungsbild der ADHS bei Erwachsenen keineswegs überraschend, da im Laufe der Zeit Kompetenz- und Performanzdefizite sowie ungünstige Lebensumstände in den Vordergrund rücken. Neuere Untersuchungen und Metaanalysen zeigen denn auch, dass die Pathogenese von ADHS bei Erwachsenen kaum mehr durch psychophysiologische oder genetische Kennwerte allein erklärt werden kann [z.B. Monastra et al., 2001; Sanchez-Mora et al., 2010].

Erwachsene mit ADHS haben einen hohen Bedarf an individuellen (Selbst-)Organisationskompetenzen, die naturgemäß über eine Medikation nicht aufgebaut werden können, 
sondern das Erlernen neuer Verhaltensstrategien voraussetzen. Die hohe Wirksamkeit eines solchen verhaltenstherapeutischen Ansatzes ist für das Kindesalter bereits metaanalytisch belegt. So errechneten Fabiano et al. [2009] unter Einbeziehung von 174 Studien je nach Evaluationsdesign diesbezügliche Effektstärken von 0,70 bis 3,78.

Der gegenwärtige Stand der Wirksamkeitsforschung zeichnet sich zurzeit noch durch eine Fülle von Schwächen aus, die möglichst rasch behoben werden müssen. So liegen zum einen noch zu wenige Studien vor. Dies dürfte auch dem Umstand geschuldet sein, dass psychologische Konzepte der ADHSPsychotherapie im Erwachsenenalter erst seit einigen Jahren entwickelt und evaluiert werden. Unbefriedigend ist, dass die Studien zur Wirksamkeit psychotherapeutischer Behandlungen zumeist nicht kontrolliert-randomisiert sind. Zudem erzielen die häufig durchgeführten reinen Prä-post-Vergleiche (8 von 12 Psychotherapiestudien) leichter höhere Effektstärken als Studien, die Kontrollengruppen mit einbeziehen (4 Psychotherapiestudien).

Die vorliegende Metaanalyse belegt auch deutliche Unzulänglichkeiten in der Definition der Störung selbst. Sie wird keineswegs nach einem einheitlichen Standard definiert. Zwar wird in den einzelnen Studien Bezug genommen auf die bestehenden differentialdiagnostischen Klassifikationssysteme, die zu den Stichprobenzuweisungen führten, jedoch werden weder in der ICD-10 (International Classification of Diseases, World Health Organization [Dilling et al., 2004]) noch im DSM-IV-TR (Diagnostic and Statistical Manual of Mental Disorders, American Psychiatric Association [Saß et al., 2003]) Kriterien für das Erwachsenenalter angeführt. Die Kriterien der Kindheit treffen auf das Erwachsenenalter aber nur sehr begrenzt zu, da sich die Störungssymptomatik verändert. Es ist eine retrospektive Beurteilung erforderlich, ob die Symptome in Kindheit und Jugend durchgehend erfüllt waren [McGough und Barkley, 2004]. Speziell für Erwachsene liegen die Wender-Utah-Kriterien der Aufmerksamkeitsdefizit-/ Hyperaktivitätsstörung des Erwachsenenalters vor [Retz-Junginger et al., 2002], bei denen 7 Symptomcluster der adulten ADHS genannt werden. Die Einbeziehung derart spezifischer Kriterien ist jedoch kaum erfolgt.

Zudem werden in den verschiedenen Studien ganz unterschiedliche Maße und Prozeduren heranzogen. So fällt die Homogenitätsprüfung bezüglich der Pharmakastudien signifikant aus, was darauf hinweist, dass die den Studien zugrunde liegenden Populationen stark divergieren.

In den einzelnen Studien werden oft sehr verschiedene Erfolgsmaße herangezogen. Sie reichen von Ratingskalen zur ADHS-Symptomatik (etwa ADHD-Rating-Scale-IV, Wender-Reimherr Adult Attention Deficit Disorder Scale, Conner's Adult ADHD Rating Scale), Depressionsinventa- ren, Messverfahren zum Ärgerausdruck und Angstskalen bis zur Erfassung von Leistungen und Funktionsfähigkeit im Alltag (Global Assessment of Functioning-Scale, Wortflüssigkeit, Intelligenz). Teilweise werden sogar bis zu 9 Gruppen abhängiger Variablen mit wiederum mehreren Untertests erfasst [Hesslinger et al., 2002]. Es wird deutlich, dass es eine Einigung darüber geben sollte, wie der Erfolg einer Therapie bei ADHS sinnvollerweise gemessen werden kann. Gemäß der derzeitigen Befundsituation eignen sich insbesondere 2 Gruppen abhängiger Variablen dafür, und zwar jene, die erstens die Reduzierung der Symptomatik und zweitens die Verbesserung von Alltagshandeln erfassen. Zu Ersterem liegen mehrere ADHS-spezifische Symptomlisten vor, zu Letzterem empfehlen sich Maße zur Zielerreichung und zur Funktionsfähigkeit im Alltag.

Allerdings ist eine komplette methodische Standardisierung von Therapie und Erfolgsmessung in diesem heterogenen Forschungsfeld auch nicht uneingeschränkt zu wünschen, da spezifische Problemkonstellationen (beispielsweise ADHS kombiniert mit Suchtverhalten oder sozialen Ängsten) individualisierte Therapieplanungen erfordern.

Es ist jedoch ein formaler Standard zu fordern, der eine präzise Stichprobenselektion, die Notwendigkeit zu Randomisierungen und von Kontrollgruppen sowie die Definition von zentral bedeutsamen Erfolgskriterien (vor allem ADHSSymptomatik) berücksichtigt.

Aktuell untersucht die Forschergruppe des Förderprojekts «Forschungsverbünde zur Psychotherapie» des Bundesministeriums für Bildung und Forschung (BMBF) Wirkung und Wirkmechanismen der Psychotherapie zur Behandlung der ADHS bei Kindern und Erwachsenen und formuliert einen anspruchsvollen methodischen Standard der Psychotherapieforschung, der effekt- und teststärkenbezogene Bestimmung der Stichprobengröße, Randomisierung, Kontrollbehandlung, manualisierte Psychotherapie, Sicherstellung der Manualtreue, blindes Rating des Behandlungserfolgs und Intentionto-treat-Analyse umfasst [Philipsen et al., 2009].

Ein beträchtlicher Bedarf besteht nach Wirksamkeitsstudien über kombinierte Behandlungen, zumal in der Praxis diese Kombination tatsächlich häufig erfolgt.

Insgesamt wird deutlich, dass Interventionen bei ADHS im Erwachsenenalter bei Weitem noch nicht so tiefgehend evaluiert worden sind wie entsprechende Interventionsmaßnahmen bei Kindern und insofern hier noch dringender Nachholbedarf besteht.

\section{Disclosure Statement}

Es liegt kein Interessenkonflikt im Sinne der allgemeinen Richtlinien vor. 


\section{Literatur}

Adler L, Liebowitz M, Kronenberger W, Qiao M,

Rubin R, et al.: Atomoxetine treatment in adults with attention-deficit/hyperactivity disorder and comorbid social anxiety disorder. Depress Anxiety 2009a:26:212-221.

Adler L, Spencer T, Brown T, Holdnack J, Saylor K, et al.: Once-daily atomoxetine for adult attentiondeficit/hyperactivity disorder: a 6-month double-

blind trial. J Clin Psychopharmacol 2009b;29:44-50.

Adler LA, Spencer T, McGough JJ, Jiang H, Muniz R:

Long-term effectiveness and safety of dexmethylphenidate extended-release capsules in adult ADHD. J Atten Disord 2009c;12:449-459.

Aron A, Dowson J, Sahakian B, Robbins T: Methyl-

phenidate improves response inhibition in adults with attention-deficit/hyperactivity disorder. Biol Psychiatry 2003;54:1465-1468.

Barkley RA, Murphy KR, Fischer M: ADHD in Adults: What the Science Says. New York, Guilford Press, 2008.

Barton J: Atomoxetine: a new pharmacotherapeutic approach in the management of attention deficit/ hyperactivity disorder. Arch Dis Child 2005;90 (suppl 1):26-29.

Biederman J, Mick E, Surman C, Doyle R, Hammer-

ness P, et al.: A randomized placebo-controlled tria of OROS methylphenidate in adults with attentiondeficit/hyperactivity disorder. Biol Psychiatry 2006; 59:829-835.

Bouffard R, Hechtman L, Minde K, Iaboni-Kassab F The efficacy of 2 different dosages of methylphenidate in treating adults with attention-deficit hyper-

activity disorder. Can J Psychiatry 2003;48:546-554

Bramham J, Young S, Bickerdike A, Spain D, McCar$\tan \mathrm{D}$, Xenitidis K: Evaluation of group cognitive behavioral therapy for adults with ADHD. J Atten Disord 2009:12:433-441.

British Medical Association und The Royal Pharmaceutical Society of Great Britain: British Nationa Formulary (BNF) 54. London, British Medical Association und Royal Pharmaceutical Society of Great Britain, 2007.

Carpentier P, de Jong C, Dijkstra B, Verbrugge C,

Krabbe P: A controlled trial of methylphenidate in adults with attention deficit/hyperactivity disorder and substance use disorders. Addiction 2005;100: 1868-1874.

Castells X, Ramos-Quiroga JA, Rigau D, Bosch R,

Nogueira M, Vidal X, Casas M: Efficacy of methylphenidate for adults with attention-deficit hyperactivity disorder: a meta-regression analysis. CNS Drugs 2011;25:157-169.

Conners C, Levin E, Sparrow E, Hinton S, Erhardt D,

et al.: Nicotine and attention in adult attention deficit hyperactivity disorder (ADHD). Psychopharmacol Bull 1996;32:67-73.

Dilling H, Mombour W, Schmidt MH: Internationale Klassifikation psychischer Störungen. ICD-10 Kapitel V (F). Diagnostische Kriterien für Forschung und Praxis, ed 3. Bern, Huber, 2004

Ebert D, Krause J, Roth-Sackenheim C: ADHS

im Erwachsenenalter: Leitlinien auf der Basis eines Expertenkonsensus mit Unterstützung der DGPPN. Nervenarzt 2003;74:939-946.

Fabiano GA, Pelham WE, Coles EK, Gnagy EM,

Chronis-Tuscano A, O'Connor BC: A meta-analysis of behavioral treatments for attention-deficit/ hyperactivity disorder. Clin Psychol Rev 2009;29: 129-140.

Faraone SV, Glatt SJ: A comparison of the efficacy of

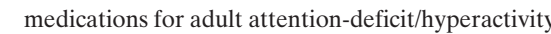
disorder using meta-analysis of effect sizes. J Clin Psychiatry 2010;71:754-763.
Faraone SV, Spencer T, Aleardi M, Pagano C, Biederman J: Meta-analysis of the efficacy of methylphenidate for treating adult attention-deficit/hyperactivity disorder. J Clin Psychopharmacol 2004;24:24-29.

Fayyad J, De Graaf R, Kessler R, Alonso J, Angermeyer M, et al.: Cross-national prevalence and correlates of adult attention-deficit hyperactivity disorder. Br J Psychiatry 2007;190:402-409.

Halperin JM, Schulz KP: Revisiting the role of the

prefrontal cortex in the pathophysiology of attention-deficit/hyperactivity disorder. Psychol Bull 2006;132:560-581.

Hesslinger B, van Elst LT, Nyberg E, Dykierek P,

Richter H, et al.: Psychotherapy of attention deficit hyperactivity disorder in adults: a pilot study using a structured skills training program. Eur Arch Psychiatry Clin Neurosci 2002;252:177-184.

Jain U, Hechtman L, Weiss M, Ahmed TS, Reiz JL, et

$\checkmark$ al.: Efficacy of a novel biphasic controlled-release methylphenidate formula in adults with attentiondeficit/hyperactivity disorder: results of a doubleblind placebo-controlled crossover study. J Clin Psychiatry 2007;68:268-277.

Kinsbourne M, De Quiros G, Rufo D: Adult ADHD

- controlled medication assessment. Adult Atten Defic Disord 2001;931:287-296.

Koesters M, Becker T, Kilian R, Fegert JM, Wein-

mann S: Limits of metaanalysis: methylphenidate in the treatment of adult attention-deficit hyperactivity disorder. J Psychopharmacol 2009;23:733-744.

Kooij J, Burger H, Boonstra A, Van der Linden P,

Kalma L, Buitelaar J: Efficacy and safety of methylphenidate in 45 adults with attention-deficit/hyperactivity disorder: a randomized placebo-controlled double-blind cross-over trial. Psychol Med 2004;34: 973-982.

Kuperman S, Perry PJ, Gaffney GR, Lund BC, BeverStille KA, et al.: Bupropion SR vs. methylphenidate vs. placebo for attention deficit hyperactivity disorder in adults. Ann Clin Psychiatry 2001;13:129-134.

Laucht M, Skowronek MH, Becker K, Schmidt MH,

Esser G, Schulze TG, Rietschel M: Interacting effects of the dopamin transporter gene and psychosocial diversity on attention-deficit/hyperactivity disorder symptoms among 15-years-olds from a high-risk community sample. Arch Gen Psychiatry 2007;64:585-590.

Lauth G, Raven H: Aufmerksamkeitsdefizit/Hyperaktivitätsstörungen (ADHS) im Erwachsenenalter: Ein Review. Psychotherapeutenjournal 2009;1:17-30.

Lauth GW, Breuer J, Minsel WR: Goal Attainment Scaling in der Ermittlung der Behandlungswirksamkeit bei der behavioralen Therapie von Erwachsenen mit ADHS: Eine Pilotstudie. Z Psychiatr Psychol Psychother 2010;58:45-53

Lauth GW, Minsel W: ADHS bei Erwachsenen: Diagnostik und Behandlung von Aufmerksamkeits-/ Hyperaktivitätsstörungen. Göttingen, Hogrefe, 2009.

Levin E, Conners C, Silva D, Canu W, March J: Ef-

fects of chronic nicotine and methylphenidate in adults with attention deficit/hyperactivity disorder. Exp Clin Psychopharmacol 2001;9:83-90.

Levin E, Conners C, Sparrow E, Hinton S, Erhardt D,

et al.: Nicotine effects on adults with attention-deficit hyperactivity disorder. Psychopharmacology 1996;123:55-63.

Linehan MM: Cognitive Behavioral Treatment of Borderline Personality Disorder. New York, Guilford Press, 1993.

Mattes J, Boswell L, Oliver H: Methylphenidate ef-

fects on symptoms of attention deficit disorder in adults. Arch Gen Psychiatry 1984;41:1059-1063.
McGough JJ, Barkley RA: Diagnostic controversies in adult attention deficit hyperactivity disorder. Am J Psychiatry 2004;161:1948-1956.

Medori R, Ramos-Quiroga J, Casas M, Kooij J,

Niemela A, et al.: A randomized placebo-controlled trial of three fixed dosages of prolonged-release OROS methylphenidate in adults with attentiondeficit/hyperactivity disorder. Biol Psychiatry 2008, 63:981-989.

Meszaros A, Czobor P, Balint S, Komlosi S, Simon V,

Bitter I: Pharmacotherapie of adult attention deficit hyperactivity disorder (ADHD): a meta-analysis. Int J Neuropsychopharmacol 2009;12:1137-1147.

Michelson D, Adler L, Spencer T, Reimherr F, West

S, et al: Atomoxetine in adults with ADHD: two randomized placebo-controlled studies. Biol Psychiatry 2003;53:112-120.

Miller TW, Nigg JT, Faraone SV: Axis I and II comor

bidity in adults with ADHD. J Abnorm Psychol 2007:116:519-528.

Monastra VJ, Lubar FJ, Linden M: The developmen

of a quantitative electroencephalographic scanning process for attention deficit-hyperactivity disorder: Reliability and validity studies. Neuropsychology 2001:15:136-144.

National Collaborating Centre for Mental Health: Diagnosis and Management of ADHD in Children, Young People and Adults. London, The British Psychological Society and The Royal College of Psychiatrists, 2009

Paterson R, Douglas C, Hallmayer J, Hagan M,

Krupenia Z: A randomised double-blind placebocontrolled trial of dexamphetamine in adults with attention deficit hyperactivity disorder. Aust N Z J Psychiatry 1999;33:494-502.

Philipsen A, Richter H, Peters J, Alm B, Sobanski E,

et al.: Structured group psychotherapy in adults with attention deficit hyperactivity disorder. J Nerv Ment Dis 2007;195:1013-1019.

Philipsen A, van Elst LT, Lesch KP, Jans T, Warnke

A: Wirksamkeit und Wirkmechanismen von Psychotherapie bei der Behandlung der Aufmerksamkeitsdefizit-/Hyperaktivitätsstörung (ADHS) bei Kindern und Erwachsenen. Psychother Psych Med 2009;59:132-140.

Prosser B, Reid R: Psychostimulant use for children

with ADHD in Australia. J Emot Behav Disord 1999;7:110-117.

Reimherr F, Williams E, Strong R, Mestas R, Soni P,

Marchant B: Double-blind placebo-controlled crossover study of osmotic release oral system methylphenidate in adults with ADHD with assessment of oppositional and emotional dimensions of the disorder. J Clin Psychiatry 2007;68:93-101.

Reimherr FW, Hedges DW, Strong RE, Marchant

BK, Williams ED: Bupropion SR in adults with ADHD: a short-term placebo-controlled trial. Neuropsychiatr Dis Treat 2005;1:245-251.

Retz-Junginger P, Retz W, Blocher D, Weijers HG

Trott GE, Wender PH, Rössler M: Wender-Utah Rating Scale (WURS-K). Nervenarzt 2002; 73:830839.

Rösler M, Fischer R, Ammer R, Ose C, Retz W: A $\checkmark$ randomised placebo-controlled 24-week study of low-dose extended-release methylphenidate in adults with attention-deficit/hyperactivity disorder Eur Arch Psychiatry Clin Neurosci 2009;259:120 129; Erratum 2009;259:368

Rostain AL, Ramsay JR: A combined treatment approach for adults with ADHD - results of an open study of 43 patients. J Atten Disord 2006;10:150-159.

Rustenbach SJ: Metaanalyse: Eine anwendungsorientierte Einführung. Bern, Hans Huber, 2003. 
Safren SA, Otto MW, Sprich S, Winett CL, Wilens

TE, Biederman J: Cognitive-behavioral therapy for ADHD in medication-treated adults with continued symptoms. Behav Res Ther 2005;43:831-842.

Sanchez-Mora C, Ribases M, Ramos-Quiroga JA, Casas M, Bosch R, et al.: Meta-analysis of brainderived neurotrophic factor p.Val66Met in adult ADHD in four European populations. Am J Med Genet B Neuropsychiatr Genet 2010;153:512-523.

Saß H, Wittchen HU, Zaudig M, Houben I: Diagnostisches und statistisches Manual psychischer Störungen - Textrevision - DSM-IV-TR. Göttingen, Hogrefe, 2003.

Schmidt S, Petermann F: Developmental psycho-

pathology: attention deficit hyperactivity disorder (ADHD). BMC Psychiatry 2009;9:Art. 58.

Schubiner H, Saules K, Arfken C, Johanson C, Schuster $\mathrm{C}$, et al.: Double-blind placebo-controlled tria of methylphenidate in the treatment of adult ADHD patients with comorbid cocaine dependence. Exp Clin Psychopharmacol 2002;10:286-294.

Simpson D, Plosker GL: Atomoxetine: a review of

its use in adults with attention deficit hyperactivity disorder. Drugs 2004;64:205-222.

Sobanski E, Alm B: Aufmerksamkeitsdefizi-/Hyper-

aktivitätsstörung (ADHS) bei Erwachsenen: Ein Überblick. Nervenarzt 2004;75:697-716.

Solanto MV, Marks DJ, Mitchell KJ, Wasserstein J,

Kofman MD: Development of a new psychosocial treatment for adult ADHD. J Attent Disord 2008, 11:728-736.

Spencer T, Biederman J, Wilens T, Doyle R, Surman

C, et al.: A large double-blind randomized clinical trial of methylphenidate in the treatment of adults with attention-deficit/hyperactivity disorder. Biol Psychiatry 2005;57:456-463.

Spencer T, Biederman J, Wilens T, Faraone S, Prince J,

et al.: Efficacy of a mixed amphetamine salts compound in adults with attention-deficit/hyperactivity disorder. Arch Gen Psychiatry 2001;58:775-782.

Spencer T, Biederman J, Wilens T, Prince J, Hatch M,

et al.: Effectiveness and tolerability of tomoxetine in adults with attention deficit hyperactivity disorder. Am J Psychiatry 1998;155:693-695.

Spencer T, Wilens T, Biederman J, Faraone S, Ablon

J, Lapey K: A double-blind crossover comparison of methylphenidate and placebo in adults with childhood-onset attention-deficit hyperactivity disorder. Arch Gen Psychiatry 1995;52:434-443.

Spencer TJ, Adler LA, McGough JJ, Muniz R, Jiang

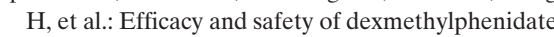
extended-release capsules in adults with attentiondeficit/hyperactivity disorder. Biol Psychiatry 2007; 61:1380-1387.
Spencer TJ, Adler LA, Weisler RH, Youcha SH: Triple-bead mixed amphetamine salts (SPD465), a novel enhanced extended-release amphetamine formulation for the treatment of adults with ADHD: a randomized double-blind multicenter placebo-controlled study. J Clin Psychiatry 2008;69: 1437-1448.

Stevenson CS, Whitmont S, Bornholt L, Livesey D,

Stevenson RJ: A cognitive remediation programme for adults with attention deficit hyperactivity disorder. Aust N Z J Psychiatry 2002;36:610-616.

Taylor F, Russo J: Comparing guanfacine and dex-

troamphetamine for the treatment of adult attention-deficit/hyperactivity disorder. J Clin Psychopharmacol 2001;21:223-228.

Taylor F, Russo J: Efficacy of modafinil compared to

dextroamphetamine for the treatment of attention deficit hyperactivity disorder in adults. J Child Adolesc Psychopharmacol 2000;10:311-320.

Taylor F, Russo J: Comparing guanfacine and dex-

troamphetamine for the treatment of adult attention-deficit/hyperactivity disorder. J Clin Psychopharmacol 2001;21:223-228.

Tenenbaum S, Paull JC, Sparrow EP, Dodd DK,

Green L: An experimental comparison of Pycnogenol ${ }^{\circledR}$ and methylphenidate in adults with attention-deficit/hyperactivity disorder (ADHD). J Atten Disord 2002;6:49-60.

Turner D, Clark L, Dowson J, Robbins T, Sahakian B:

Modafinil improves cognition and response inhibition in adult attention-deficit/hyperactivity disorder. Biol Psychiatry 2004;55:1031-1040.

Verster J, Bekker E, de Roos M, Minova A, Eijken E,

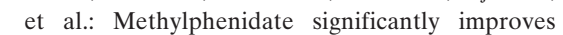
driving performance of adults with attention-deficit hyperactivity disorder: a randomized crossover trial. J Psychopharmacol 2008;22:230-237.

Virta M, Vedenpää A, Grönroos N, Chydenius E, Partinen M, et al.: Adults with ADHD benefit from cognitive-behaviorally oriented group rehabilitation. J Atten Disord 2008;12:218-226.

Volkow ND, Wang GJ, Fowler JS, et al.: Evidence that methylphenidate enhances the saliency of a mathematical task by increasing dopamine in the

human brain. Am J Psychiatry 2004;161:1173-1180.

Vorstand der Bundesärztekammer: Stellungnahme zur Aufmerksamkeitsdefizit-/Hyperaktivitätsstörung. http://www.bundesaerztekammer.de/page.asp? his=0.7.47.3161.3163.2005. Netzzugriff am 28.01.2009.

Weisler RH, Biederman J, Spencer TJ, Wilens TE,

Faraone SV, et al.: Mixed amphetamine salts extended-release in the treatment of adult ADHD: a randomized controlled trial. CNS Spectr 2006;11: 625-639.
Weiss M, Hechtman L: A randomized double-blind

trial of paroxetine and/or dextroamphetamine and problem-focused therapy for attention-deficit/ hyperactivity disorder in adults. J Clin Psychiatry 2006;67:611-619.

Wender P, Reimherr F, Wood D: Attention deficit disorder (minimal brain-dysfunction) in adults. Arch Gen Psychiatry 1981;38:449-456.

Wender P, Reimherr F, Wood D, Ward M: A control-

led-study of methylphenidate in the treatment of attention deficit disorder residual type in adults. Am J Psychiatry 1985;142:547-552.

Wiggins D, Singh K, Getz HD, Hutchins DE: Effects of brief group intervention for adults with attention deficit/hyperactivity disorder. J Ment Health Couns 1999;21:82-93.

Wilens T, Biederman J, Prince J, Spencer T, Faraone

S, et al.: Six-week double-blind placebo-controlled study of desipramine for adult attention deficit hyperactivity disorder. Am J Psychiatry 1996;153: 1147-1153.

Wilens T, Haight B, Horrigan J, Hudziak J, Rosenthal

${ }_{\mathrm{N}}$, et al.: Bupropion XL in adults with attentiondeficit/hyperactivity disorder: a randomized placebo-controlled study. Biol Psychiatry 2005;57:793801.

Wilens T, Spencer T, Biederman J, Girard K, Doyle R,

et al.: A controlled clinical trial of bupropion for attention deficit hyperactivity disorder in adults. Am J Psychiatry 2001;158:282-288.

Wilens TE, Klint T, Adler L, West S, Wesnes K, et al.

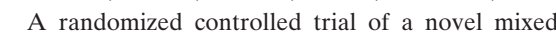
monoamine reuptake inhibitor in adults with ADHD. Behav Brain Funct 2008;4:24

Wilens TE, McDermott SP, Biederman J, Abrantes A, Hahesy A, Spencer TJ: Cognitive therapy in the treatment of adults with ADHD: a systematic chart review of 26 cases. J Cognit Psychother 1999;13: 215-227.

Wolraich ML, McGuinn L, Doffing M: Treatment of

attention deficit hyperactivity disorder in children and adolescents: safety considerations. Drug Safety 2007;30:17-26.

Wood D, Reimherr F, Wender P, Johnson G: Diagno-

sis and treatment of minimal brain dysfunction in adults - preliminary report. Arch Gen Psychiatry 1976;33:1453-1460.

Zylowska L, Ackermann DL, Yang MH, Futrell JL,

Horton NL, et al.: Mindfulness meditation training in adults and adolescents with ADHD. J Atten Disord 2008;11:737-746. 